\title{
HOSPITAL OUTBREAK OF POST CAESAREAN WOUND INFECTION WITH ATYPICAL MYCOBACTERIA
}

\author{
Battu Vijayalaxmi1, Vangala Usha Rani², Rama Saraladevi3i, Lanka Radhika ${ }^{4}$
}

${ }^{1}$ Assistant Professor, Department of Obstetrics and Gynaecology, Kakatiya Medical College, Warangal. ${ }^{2}$ Assistant Professor, Department of Obstetrics and Gynaecology, Kakatiya Medical College, Warangal. ${ }^{3}$ Associate Professor, Department of Obstetrics and Gynaecology, Kakatiya Medical College, Warangal. ${ }^{4}$ Tutor, Department of Obstetrics and Gynaecology, Kakatiya Medical College, Warangal.

\section{ABSTRACT}

Study of 18 cases of post-operative CS wound infection due to Atypical Mycobacterium (M. chelonae) were found in a single hospital over a six-month period. Though the contaminating source could not be identified, remedial control measures taken includes changing all the operative equipment, autoclave machine, air conditioners, exhaust fans of OT, linen and mattresses and solutions like betadine, savlon, spirit and also suture material replaced with new ones. Antibiotic protocols are changed and daily cleaning of OT with cetrimide solution and daily fumigation for one month. No further Atypical MB infective episode have occurred in the one and a half year since the study. Awareness of this ubiquitous opportunistic organism that is not easily eradicated from the hospital environment and strong suspicion of chronic post-operative wound infection, careful surveillance, detailed attention to disinfectant methods of medical devices and appropriate control measures are essential to prevent potential outbreaks.

\section{KEYWORDS}

Atypical Mycobacteria, Mycobacterium Chelonae, Non-Tuberculous Mycobacteria (NTM).

HOW TO CITE THIS ARTICLE: Vijayalaxmi B, Rani VU, Saraladevi R, et al. Hospital outbreak of post caesarean wound infection with atypical mycobacteria. J. Evolution Med. Dent. Sci. 2016;5(52):3416-3419, DOI: 10.14260/jemds/2016/789

\section{INTRODUCTION}

Atypical mycobacteria have been known to colonize tap water, natural waters and soil and thus can easily contaminate solutions and disinfectants used in hospital settings. These infections have thus been a source of significant morbidity for patients recovering from laparoscopic surgeries. Erroneous sterilization of instruments is almost always responsible for such outbreaks and makes it a problem mainly affecting developing countries such as India. Thus proper sterilization of such instruments is essential to prevent the occurrence of post laparoscopic wound infections with atypical mycobacteria.

There has been much controversy surrounding the proper line of treatment for port whole infections with atypical mycobacteria. These micro-organisms show limited response to first line anti-tuberculosis drugs. Thus, the standard treatment consists of combinations of second line antitubercular drugs including macrolides such as clarithromycin, quinolones such as ciprofloxacin, tetracyclines such as doxycycline and aminoglycosides such as amikacin. Once there is manifestation of clinical symptoms, the standard treatment consists of a 28-day regimen of oral clarithromycin and ciprofloxacin or amikacin. However, local administration of aminoglycosides has been shown to be highly efficacious in the treatment of particularly stubborn nodules and sinuses that persist after completion of oral therapy.

Atypical Mycobacteria also known as non-tuberculous Mycobacteria and Mycobacteria other than Mycobacterium tuberculosis complex (M. Tuberculosis, M. Microti, M. Bovis

Financial or Other, Competing Interest: None.

Submission 29-04-2016, Peer Review 08-06-2016,

Acceptance 14-06-2016, Published 30-06-2016.

Corresponding Author:

Vangala Usha Rani,

Assistant Professor,

Govt. Maternity Hospital,

Hanamkonda,

Warangal.

E-mail:vangalausha7@gmail.com

DOI: $10.14260 /$ jemds $/ 2016 / 789$ and M. africanum), environmental bacteria. These Mycobacteria have been responsible for increasing worldwide reports of hospital outbreaks and isolated cases of Atypical Mycobacterial inections. ${ }^{1-5}$

Atypical mycobacteria is classified into 4 groups. Group 1: Photochromogens are slow growing, Eg:- M. kansasii causes chronic pulmonary diseases. Man-to-Man transmission does not occur. Group 2: Scotochromogens are M. Scrofulaceum causes cervical adenitis in children. Group 3: Non-chromogens are M. Intracellulare (Pulm. TB), M. avium (Cervical adenitis, Human infections rare), M. xenopi occasionally causes chronic lung diseases. Group 4: Rapid growers are M. fortuitum, M. chelonae, both of which causes chronic abscesses in human being.

In the present study organism identified is M. chelonae belonging to group $4 \mathrm{AMB}$. These are rapidly growing $\mathrm{MB}$, widely distributed in nature having been isolated from natural water, tap water, soil and water used in showers and surgical solutions. ${ }^{6}$ They have been the cause of a variety of clinical presentations in cutaneous disease, but rarely cause disseminated infections. The most frequently reported infections are post-surgical, primary cutaneous and pulmonary. ${ }^{7-8}$ The detection and identification of causative agent may be missed unless a direct Ziehl-Neelsen (ZN) stain for acid fast bacilli and culture on Lowenstein Jensen (LJ) media is done for all chronic post-surgical wound infections, since routine aerobic cultures are sterile and antibiotics for pyogenic infections do not work satisfactorily.

These atypical mycobacteria are distributed widely in the environment and contaminate municipal water supply and are resistant to sterilizers, antiseptics and standard disinfectants. RGM, the hydrophobic organisms have the ability to form biofilms for its successful survival in the environment. Hence, these organisms are difficult to eradicate even with the regular disinfectants like alkaline glutaraldehydes, organomercurials and chlorine. Shedding of these organisms from the biofilm in a water pipe or device may be the reason for acquiring this infection. 
Improperly sterilized endoscopes and instruments thus cause post-operative wound infections and injection abscess.

The frequency of non-tuberculous mycobacterial infection is increasing worldwide. In most parts of India, there is no standardized operative procedures followed for cultures and also these organisms could be ignored as contaminants; it is not possible to determine the exact incidence of these infections. Herein, we describe case series of surgical site infections due to rapidly growing Mycobacteria seen in our hospital.

\section{CASE SERIES}

Atypical Mycobacterial infections in 18 patients following caesarean section in a single hospital over a 6 months period from April 2014 to September 2014, who presented with repeated wound site infections and discharging sinuses. Of the total 180 cases delivered in the hospital, 18 patients who underwent LSCS operations presented with recurrent wound site infections (Induration, discharging sinuses, abscesses) at the site of incision within a duration of 4 weeks to 8 weeks of their surgical procedure. Postoperatively, all the patients had apparently healthy wounds and stitches were removed between $5^{\text {th }}$ and $7^{\text {th }}$ POD and were discharged; 17 cases reported with discharging sinuses, out of which 5 cases underwent secondary suturing.

They again reported back with suture line pus discharge and discharging sinuses. All the routine cultures came out as sterile and they were treated with IV antibiotics, 3 cases underwent wound debridement and tissue sent for HPE. HPE showed Langhans cells and AFB staining positive for two. With review of literature and suspicion of atypical mycobacteria outbreak, cultures for Atypical Mycobacterium and AFB staining were sent for one patient, as she was still having discharge.

All 17 patients were afebrile and one without any constitutional symptoms; 18 patients presented with high grade fever after one month of surgery which was not responded to routine IV antibiotics and developed bilateral inguinal abscess when treated with IV Ofloxacin. Then with the help of general surgeon abscess is drained and pus is sent for routine culture, which was -ve (Sterile). Fever still continued.

Ultrasound revealed subcutaneous collection over the rectus sheath and intraperitoneal collection. Debridement planned and only fewer collections noted and not able to explain the fever. Abdomen was opened and found nothing. Closure done with bilateral subcutaneous drains. HPE of the tissue showed positive for Langhans cells with AFB +ve. Keeping HPE report in view, discharge from drain wound sent for culture for Atypical Mycobacteria, which was +ve for M. chelonae, PCR TB positive for this patient. Taken the entire events round in April to July, outbreak of ATM suspected. The discharge sinuses from the other patients could not be cultured for Atypical Mycobacteria, because they were near dry except for small holes in the suture line.

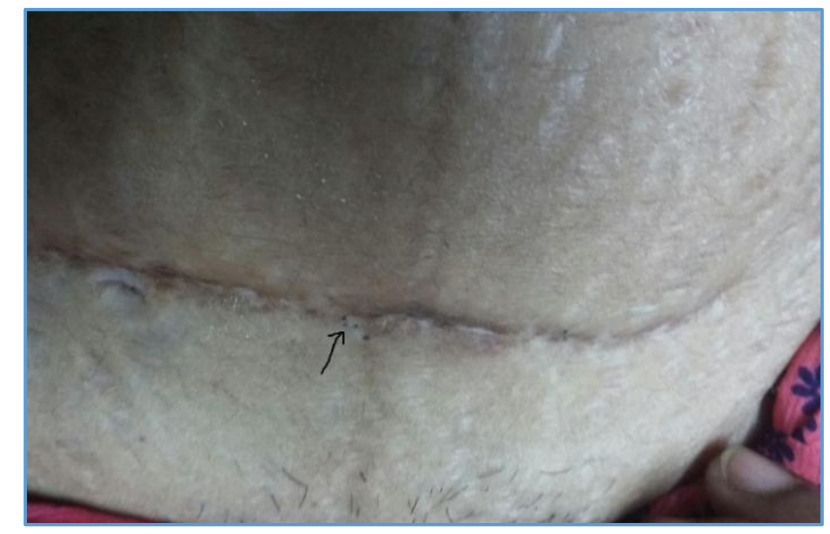

Fig. 1: Postoperative Wound showing Sinuses

All these patients were called back, counselled and treated with combination of 3 drug regimen of antibiotics (Moxiflox, clarithromycin, Azithromycin, Linezolid, Ofloxacin, Amikacin, Ethambutol, ATT) for 6 weeks to 3 months period depending on severity of infection with guidance of infection disease specialist.

After taking the control measures and changing the antibiotic protocols ( 3 drug regimen), no case was reported for one and a half year since the study. Postoperative wound showing sinuses. Once the causative agent was identified, hospital policy regarding disinfection was reviewed. Hospital was closed. After consultation with microbiologist, all the water sources in hospital (Tap water, Tank water) sent for culture. Swabs from all the walls and floor of theatre, wards and labour room sent for culture. Culture came out -ve for M. chelonae and AFB.

All the equipment, linen, autoclave and air conditioners, Boyle's, $\mathrm{O}_{2}$ masks, exhaust fans, mattress from hospital were replaced with new ones. All the stock in hospital like IV fluid, savlon, betadine solution, spirit and suture material were thrown out and replaced with new ones. Antibodies protocols were changed. Daily fumigation of hospital was done. Hospital and theatre were daily treated with $2 \%$ cetrimide from roof to floor and walls for 1 month. Again swabs sent, which came out -ve. Hospital reopened in November, from then no case was reported. Microbiological Investigations: Purulent material of 0.5-2.0 mL aspirate was collected with sterile syringe or two swabs from the wound site were processed for the identification of the causative agents. In addition, in herniotomy patients, mesh was also sent. Gram, Ziehl-Neelsen and lactophenol cotton blue stains were done for bacteria, mycobacteria and fungi respectively. They were then inoculated in blood, MacConkey, chocolate, Sabouraud dextrose agar and Lowenstein-Jensen medium for aerobic bacteria, fungi and mycobacteria respectively. Culture was also put on Robertson cooked meat media to exclude anaerobes. They were incubated at $37^{\circ} \mathrm{C}$. The isolated organisms were identified as per the standard bacteriological techniques. The growths on LJ media were further processed for additional tests as shown in to confirm the identity of the organism. Antibiotic sensitivity testing was done by Kirby Bauer method for amikacin, ciprofloxacin, clarithromycin, rifampicin, tobramycin and linezolid.

\section{DISCUSSION}

Rapidly growing mycobacteria have emerged as significant human pathogens, causing various infections in healthy and immunocompromised hosts. In conditions exposed to 
contamination of the wound with disinfectants, soil and water solution. These organisms have been increasingly reported in the past three decades in post-surgical and post-traumatic wound infections and lately increased incidence in localized and disseminated infections including outbreaks due to contaminated instruments have been observed. Postoperative wound infections caused by RGM generally appear some weeks to some months following the procedure. Similarly, in our case series the incubation period ranged from 20 to 66 days with a median incubation period of 42 days. On the contrary infections due to other pyogenic bacteria have a shorter incubation period as compared to RGM, which have a longer incubation period ranging from several days to several months. The absence of clinical response after the administration of antimicrobial agents against commonly invading bacteria (e.g. Staphylococci, Streptococci) and the sterility of routine cultures taken from the infected sites were clues for our infection.

The median time between the onset of symptoms and the microbiological diagnosis was 104 days. Therefore, a high index of suspicion is imperative for the diagnosis to be made. A study by Joon Young Song et al, stated that since the symptoms are relatively mild and indolent, the clinical diagnosis of mycobacteriosis is often delayed and took more than two months from initial manifestation to diagnosis. Also, in the revised literature, most publications conclude that clinical diagnosis of mycobacterial skin and soft tissue infections is not easy to perform and that the diagnosis is often delayed. A high degree of clinical suspicion and appropriate microbiological techniques are necessary to avoid delays in diagnosis.

Clinically, the infections caused by RGM in post-operative wound infections are similar to pyogenic abscesses within duration, microabscess and discharge from sinuses and erythema. Systemic manifestations like fever and chills are rare. The clinical features in our study were also similar with erythematous nodules, indurations, microabscesses and discharging sinuses. All our patients presented with only local manifestations that started with painful nodules which gradually increased in size, which then would fistulise and open on the skin draining pus, while none of them had any systemic manifestations.

All our patients with postoperative wound infections had repeated sterile aerobic and anaerobic cultures. Hence, it is very difficult to clinically diagnose infections, because they lack characteristic clinical features. Surgical site infections with abscess and chronic inflammation should be initially managed with conventional antibiotics; however, if there is no response and gram stain and routine culture are negative then Mycobacterial infection should be strongly suspected. Hence, AFB staining and culture for Mycobacterium should be done for sterile culture.

Though non-tubercular mycobacteria infections cause little mortality, they can cause morbidity, especially when they are not diagnosed and therefore not treated effectively. NTM are important human pathogens and have been reported from postsurgical wound infections in many countries including India. ${ }^{9}$ Breast implant infection and $\mathrm{M}$. fortuitum group was reported by Vinh et $\mathrm{al}^{10}$ In our study, all 18 cases with postoperative Surgical Site Infection [SSI] had a sterile aerobic and anaerobic cultures. Typically, wound infections due to atypical mycobacteria do not occur as an immediate post-operative complication. ${ }^{9}$ There is apparent immediate post-operative healing and gradually over a variable period of time. The scar breaks down to a persistent non-healing superficial wound with discharging sinuses, as also in our study where 17 of these patients had presented with discharge between 4 and 8 weeks postoperatively. Such wounds do not respond to antibiotics used for acute infections and persist for prolonged period of time. The wounds were painless and all the patients except one are afebrile with no other systemic illness. A chronic non-healing wound may therefore present a confusing picture and in such cases Mycobacterial infection should always be ruled out by appropriate investigations, specially AFB staining.

Water, soil, animals and marine life have been mentioned as sources of M. fortuitum-chelonae complex. ${ }^{9}$ as the sample collection with stringent criteria is still not routinely followed in most parts of India and there is tendency to ignore such isolates as contaminants. It would therefore be difficult to comment on the exact magnitude of problem. Further studies to reveal the sources of infection will also be helpful in the epidemiologic control of such infections. ${ }^{9}$ PCR has been used to aid in diagnosing these conditions. In our study, we isolated M. chelonae and PCR +ve in one case.

SSI due to M. fortuitum are well documented, especially in association with CT surgery. ${ }^{11}$ Delayed wound healing, chronicity of infection with prolonged course of expensive antibiotics makes it a serious nosocomial infection. The source is frequently contamination of the wound directly or indirectly with colonized tap water. Surveillance environmental culture from water sources, basin and OT may not yield the growth of NTM. Other nosocomial infection with this organism include infection of implanted devices and injection site abscesses. Strict sterilization of OT equipment and proper hand washing must be undertaken to prevent wound infections. ${ }^{9}$

As most of atypical MB are not sensitive to routine antitubercular therapy, it is important to identify the causative MB. Diagnosis of M. chelonae infection is difficult and time consuming. So we must follow the protocol of subjecting any discharge smear studies including ZN stain, so that it may permit identification of AFB as early as possible.

The prevention of nosocomial infection and pseudo infections due to non-tuberculous MB is challenging. Their high lipid content, triple layered cell wall render mycobacteria more resistant to killing by disinfection, elevated temperature and UV light compared with other pathogenic bacteria. In addition, biofilms appear to support MB growth and protect the organism, which makes complete disinfection of colonized water systems difficult to achieve. ${ }^{12}$ Infection with NTM can be treated with a variety of antibiotics, even though first line antiTB drugs like ethambutol and rifampicin have a cidal effect against the organism, they are not used commonly. The preferred choice or a varying combination of antibacterial agents like amikacin, fluorinated quinolones, imipenem and clarithromycin. ${ }^{13}$

\section{CONCLUSION}

Non-TB MB are unique not only in their in-vitro cultivation characteristics, but also in clinical presentations. Predominant presentations include post-operative, post-infection or posttrauma wound infections and catheter associated sepsis. Three species are responsible for vast majority of diseases due to NTM: M. fortuitum, M. chelonae, M. abscesses. In India few 
cases of M. fortuitum and chelonae complex infections are being underreported due to low suspicion of its presence amongst clinicians and microbiologists. High degree of suspicion is needed for specific identification of pathogen, especially in cases of chronic postop wound infections.

Suggestive clinical features, poor response to antibiotic treatment and repeated isolation of organism from clinical specimen can help in establishing correct diagnosis and instituting prompt treatment will reduce the morbidity.

\section{REFERENCES}

1. Kotach VM. Infections due to non-tuberculous mycobacteria (NTM). Indian J Med Res 2004;120: 290-304.

2. Philips MS, von Reyn CF. Nosocomial infections due to non-tuberculous mycobacteria. Clin Infect Dis 2001;33(8):1363-74.

3. Wallace RJ, Brown BA, Griffith DE. Nosocomial outbreaks/pseudo-outbreaks caused by nontuberculous mycobacteria. Annu Rev Microbial 1998;52:453-90.

4. Covert TC, Rodger MR, Rayes AL, et al. Occurrence of nontuberculous mycobacteria in environmental samples. Appl Environ Microbial 1999;65(6):2492-6.

5. Falkinham JO. Epidemiology of infection by nontuberculous mycobacteria. Clin Microbial Rev 1996;9(2):177-215.
6. Kazda JF. The principles of ecology of mycobacteria. In: Stanford JL, Ratledge C, editors. Biology of mycobacteria. Academic Press 1983;2:323-42.

7. Brown-Elliot BA, Wallace RJ. Clinical and taxonomic status of pathogenic non-pigmented or late pigmenting rapidly growing mycobacteria. Clin Microbial Rev 2002;15(4):716-46.

8. Rotman DA, Blauvelt A, Kerdel FA. Widespread primary cutaneous infection with mycobacterium fortuitum. Int J Dermol 1993;32(7):512-4.

9. Shah AK, Gambhir RP, Hazra N, et al. Non-tuberculous mycobacteria in surgical wounds-a rising cause of concern? Indian J Surg 2010;72(3):206-10.

10. Vinh DC, Rendina A, Turner R, et al. Breast implant infection with mycobacterium fortuitum group: report of case and review. J Infect 2006;52(3):e63-7.

11. Hoffman PC, Fraser DW, Robicsek F, et al. Two outbreaks of sternal wound infection due to organisms of the M fortuitum complex. J Infect Dis 1981;143(4):533-42.

12. Vijayaraghavan R, Chandrashekhar R, Sujatha $Y$, et al. Hospital outbreak of atypical mycobacterial infection of port sites after laparoscopic surgery. Journal of Hospital Infection 2006;64(4):344-7.

13. Lahiri CKK, Jena BJ, Pannicker LCKK. Mycobacterium fortuitum infections in surgical wounds. MJAFI 2009;65:91-2. 